\title{
A Window into the Breeding Ecology and Molt of the Eastern Black Rail (Laterallus jamaicensis jamaicensis)
}

Christine E. Hand ( $\square$ handc@dnr.sc.gov )

South Carolina Department of Natural Resources https://orcid.org/0000-0002-9055-6736

\section{Wray Gabel}

South Carolina Department of Natural Resources

Gabriella R. DiPetto

South Carolina Department of Natural Resources

Rachel E. Bonafilia

South Carolina Department of Natural Resources

\section{Elizabeth Znidersic}

Institute for Land, Water and Society, Charles Sturt University

\section{Research Article}

Keywords: Black Rail, breeding ecology, camera trapping, courtship, Laterallus jamaicensis, life history, parental care, phenology, molt, Rallidae

Posted Date: June 17th, 2021

DOI: https://doi.org/10.21203/rs.3.rs-618539/v2

License: (c) (i) This work is licensed under a Creative Commons Attribution 4.0 International License. Read Full License

Version of Record: A version of this preprint was published at Waterbirds on December 23rd, 2021. See the published version at https://doi.org/10.1675/063.044.0208. 


\section{Abstract}

Knowledge of the ecology and behavior of the Eastern Black Rail (Laterallus jamaicensis jamaicensis) has remained nearly as elusive as the rail itself. Camera trapping methods facilitated the first study of breeding phenology and chick development ( $n=33$ broods), flightless molt phenology and duration ( $n=$ 10 adults in molt), and courtship and brood rearing behaviors. In addition, behavioral observations combined with phenology data provided evidence of pairs raising two or more broods during a breeding season. Conservation and management efforts for the Eastern Black Rail should take into consideration periods of vulnerability such as incubation, brood rearing and flightless molt, which coincide with increasingly severe and frequent coastal flooding events and hurricanes. To effectively develop conservation strategies to ensure the persistence of the Eastern Black Rail, it is necessary to understand factors key to the fecundity and survival of the subspecies.

\section{Main Text}

Breeding phenology includes the timing of breeding behaviors such as arrival on breeding grounds, courtship and copulation, egg laying and incubation, chick rearing, and fledging (Dunn and Moller 2014). However, in lesser studied species, these basic biological requirements often remain unknown. Rails are one of the most poorly represented groups in ornithological research (Brambilla and Jenkins 2009; Stermin et al. 2012). Ripley (1977) remarked that very little is known about the habits of the Black Rail and of species within the genus Laterallus, collectively, due to their tiny size and proclivity for secretiveness. Two subspecies of Black Rail are known to occur in the United States; Laterallus jamaicensis coturniculus (California Black Rail) in California and Arizona, and Laterallus jamaicensis jamaicensis (Eastern Black Rail) in states east of the Rocky Mountains (Eddleman et al. 2020).

The Eastern Black Rail was proposed for federal listing as a Threatened species under the Endangered Species Act as of 2018 (Endangered and Threatened Wildlife and Plants; 12-Month Petition Finding and Threatened Species Status for Eastern Black Rail with a Section 4(d) Rule 2018), and the subspecies is likely to be extirpated within the next 50 years under current conditions (U.S. Fish and Wildlife Service 2018). Conservation efforts to prevent extirpation and promote reproductive success require an understanding of phenology, fecundity, and survival; information which is scarce throughout the range of the Eastern Black Rail.

Our objectives were to document the phenology of nesting, brood rearing, and definitive prebasic molt, during which adult rails lose all remiges and retrices simultaneously, becoming temporarily flightless (hereafter flightless molt; Pyle 2008). Nesting and molting are two periods during which Black Rails have high energy requirements, are less able to move among wetlands, and may be particularly vulnerable (U.S. Fish and Wildlife Service 2018). In addition to achieving the primary objective, this study describes previously undocumented aspects of Black Rail ecology and behavior, which is a step toward filing the gaps in our knowledge of Black Rail biology. 


\section{Methods}

Study Area

We studied the breeding ecology of the Black Rail on three properties in coastal South Carolina: the Bear Island Wildlife Management Area (BIWMA; $32^{\circ} 35^{\prime} \mathrm{N}, 80^{\circ} 27^{\prime} \mathrm{W}$ ), a nearby property under private ownership, and the Tom Yawkey Wildlife Center (YWC; 3314' N, 79 $16^{\prime} \mathrm{W}$ ). BIWMA and the private property are located in Colleton County approximately $50 \mathrm{~km}$ southwest of Charleston, South Carolina in the core of the ACE (Ashepoo, Combahee, and Edisto rivers) Basin. YWC is located in Georgetown County approximately $80 \mathrm{~km}$ northeast of Charleston on islands at the confluence of the South Santee and North Santee Rivers, Winyah Bay, and the Atlantic Ocean. BIWMA (4,800 ha) and YWC (9,700 ha) are owned and managed by the South Carolina Department of Natural Resources.

Due to their cryptic and secretive nature, Black Rail presence is commonly determined using call-response surveys (Legare et al. 1999; Conway et al. 2004). Based on surveys conducted throughout the South Carolina coastal plain in 1991-1992 (Cely et al. 1993), 2014 (Roach and Barrett 2015) and 2015-2016 (Hand 2016), Black Rails frequented the impounded brackish marsh wetlands (hereafter impoundments) and salt and brackish tidal marshes (hereafter tidal marshes) at BIWMA and YWC. Impoundments at the study sites are managed using water level manipulation and prescribed burning.

We selected five wetlands (four impoundments and one tidal marsh) where Black Rails were consistently detected during call-response surveys. Within these wetlands, we identified potential Black Rail breeding territories based on microhabitat characteristics (Eddleman et al. 2020; Spautz et al. 2005) and opportunistic auditory and visual observations of Black Rails. Occupied microhabitat within the study sites was characterized by very shallow water (typically $0.5-5 \mathrm{~cm}$ ) and dense emergent vegetation. Dominant species included clump cordgrass (Spartina bakeri), saltmarsh bulrush (Schoenoplectus americanus/robustus), and narrowleaf cattail (Typha angustifolia; Wetlands 1 and 3), or smooth cordgrass (Spartina alterniflora), saltmeadow cordgrass (Spartina patens) and black needlerush (Juncus roemerianus; Wetlands 4 and 5) in impoundments or marsh fimbry (Fimbristylis castanea) and black needlerush (Wetland 2) in tidal marshes. All wetlands flooded above $20 \mathrm{~cm}$ numerous times during the study resulting from heavy rainfall (both habitat types), high tides (tidal marsh), and water level management (impoundments). The majority of Wetland 1 was burned on 28 February 2018 and again on 26 February 2019 during prescribed fires.

Motion-activated Camera Traps

To determine the phenology of breeding and flightless molt, we deployed and maintained arrays of four or more motion-activated camera traps (hereafter referred to as camera traps) within the potential/suspected territories, as described by Hand et al. (2019) during 2017-2019. Breeding by Eastern Black Rails occurs during the spring and summer and flightless molt follows the breeding season. Sampling was typically initiated during February or March and concluded during August or September due to recurring hurricanes during each year of the study. A subset of camera trap arrays that had 
consistent Black Rail detections were redeployed within one month following the storms and maintained year round. In addition to data collected during the study period, we interpreted and included brood and molt records collected during our pilot study (Hand et al. 2019).

Building upon the methods developed during our pilot study (Hand et al. 2019), we used Reconyx HyperFire Professional Covert IR camera traps (Models PC900 and HP2X) supplemented and paired with additional covert infrared camera traps with video and audio recording capability (Advanced Security SSC-24C36, Bushnell NatureView119439, 119440, and 119740, Bushnell Trophy Aggressor 119777C and 119877, and Reconyx UltraFire XP9). Reconyx Hyperfire camera traps were programmed to take ten photographs per trigger during 2017 and 5 photographs per trigger during 2018-2019. Supplemental camera traps took three photographs followed by a $10-60$ second video recording per trigger. Camera traps were programed to operate 24 hours per day with no quiet periods, and Reconyx Hyperfire camera traps were programed to take a timelapse photo every four hours in addition to motion-triggered photographs.

To maximize Black Rail detections, and due to the descriptive nature of the study, array locations and camera trap locations within arrays were not randomized or stratified. Instead, the placement of camera traps was deliberately biased (Meek et al. 2014). Locations were selected based on the presence of ideal microhabitat features within the wetland and previous observations of Black Rail movements through similar types of habitat. Within arrays, Reconyx HyperFire camera traps ( $\bar{x}=5.6 \pm 2.8 \mathrm{SD})$ were deployed $5-10 \mathrm{~m}$ apart and any supplemental camera traps $(\bar{x}=1.9 \pm 1.7 \mathrm{SD})$ were deployed $0.5-15 \mathrm{~m}$ from Reconyx HyperFire camera traps. All camera traps in the array were within a $100 \mathrm{~m}$ radius from the array center. Black Rail detections from arrays spaced $<100 \mathrm{~m}$ apart were combined because the territories of individual rails appeared to encompass portions of multiple arrays. Camera traps were mounted between $0.15 \mathrm{~m}$ and $0.75 \mathrm{~m}$ above the ground and aimed toward naturally occurring trails or gaps in clumps of vegetation, which Black Rails use to navigate the wetland (Weske 1969). Camera traps were occasionally relocated within and among arrays throughout the duration of the study to optimize Black Rail detection as water levels and suitable vegetation structure fluctuated.

We used CPW Photo Warehouse (Newkirk 2016) to organize and review photographs and to perform data summarizations. Photographs of Black Rails were categorized by sex and age when possible based on plumage characteristics (Eddleman et al. 2020; Pyle 2008).

\section{Results}

Black Rails were photographed in $86 \%$ of arrays $(n=14$, Table 1$)$ and $80 \%$ of wetlands $(n=4)$ during 2017 - 2019. Sampling effort during this period totaled 46,033 camera trap days and resulted in a total of 2,040,536 photographs, 30,841 (1.5\%) of which contained Black Rails.

Brood Size 
In broods documented within six days of hatching, brood sizes ranged from a minimum of one chick to seven chicks $(\bar{x}=3.4 \pm 1.8 \mathrm{SD}, n=16)$. For chicks $>$ ten days old when initially detected, we did not estimate brood size because chicks ventured farther from parents and siblings after frequent brooding was no longer required.

\section{Chick Development}

We documented the rate of chick development and characterized key physical and behavioral developmental stages (Tables 2 and 3 ) of broods photographed repeatedly between hatching and fledgling. Age was calculated based on chicks remaining in the nest for approximately 24 hours (Davidson 1992) and the developmental stage of the chicks photographed. Fledging occurred at approximately 40 days after hatching. In several instances, development within broods was asynchronous, resulting in a difference in fledging dates as great as three days among siblings.

\section{Breeding Phenology}

Black Rail chicks were photographed between May and September during 2015 - 2019 (Fig. 1). Estimated hatch dates ranged from 13 May -20 August ( $\bar{x}=1$ July \pm 30.0 SD, $n=33$; Table 4), suggesting incubation occurred between April and August based on an approximated 26 day period between the initiation of egg laying and hatching (Flores and Eddleman 1993; Legare and Eddleman 2001; U.S. Fish and Wildlife Service 2018). In arrays where vegetation growth was delayed by fire during February, the mean estimated hatch date was 18 July ( $\pm 28.1 \mathrm{SD}, n=9)$ compared to 25 June $( \pm 30.8 \mathrm{SD}$, $n=24)$ in arrays not burned. At least one chick survived to 15 days post-hatching in a minimum of 30 broods, and fledging was confirmed for eight broods. We did not determine if chicks from the remaining 25 broods fledged, however based on hatch dates and a period of 40 days between hatching and fledging, we estimated potential fledging dates ranged from 23 June to 30 September ( $\bar{X}=10$ August). Fledglings were photographed on 27 June 2017, between 16 July and 7 September 2018, and between 11 August and 7 October 2019.

Flightless Molt Phenology

Flightless molt (Fig. 2) was documented in Wetlands 1 and 2 during the 2015 - 2016 pilot study and in Arrays 1a, 1b and 2d in 2018 and Arrays $1 \mathrm{~b}$ and 2a during 2019. The duration of molt was approximately 21 days from the initiation of primary feather loss to the completion of primary feather regrowth. We observed ten flightless adults. In the three instances when pairs were documented molting, the paired adults molted nearly simultaneously with the females beginning two to three days later compared to males. Molt initiation dates ranged from 15 August to 20 September $(\overline{\mathrm{x}}=30$ August \pm 14.0 $\mathrm{SD}, n=10$; Table 5), with estimated molt completion between 5 September and 11 October.

Behavioral Observations

Courtship behaviors (Fig. 3) included offering food ( $n=11$ male to female, $n=1$ female to male), allopreening ( $n=7$ male preening female, $n=1$ female preening male), and pursuit of the female by the 
male $(n=25)$. Throughout the study, we documented seven copulations. During one copulation, adult male and female Black Rails were simultaneously photographed and filmed (Fig. 4). Concluding copulation, the male tumbled from the female and circled her while walking with a lowered head and raised wings. As the male circled, the female bowed forward with raised tail feathers then ruffled her feathers before beginning to preen. Throughout the study, this raised wing display by males was observed following three copulations and females were observed ruffling feathers and preening following four copulations. Males closely pursued females prior to five copulations.

In seven instances during the study, two or more broods were photographed in the same array during a single breeding season. Copulations occurred while young chicks were present in arrays during two such instances. Following copulation on 5 June 2017 in Array 2a, described above, adults departed then returned to the same location 53 minutes later with downy chicks. On 18 July 2017, downy chicks from a second brood were documented in the array. Estimated hatch dates for the first and second broods were 41 days apart. The estimated hatch date for a third brood in the array was 34 days after the hatch date for the second brood.

During 2019, four broods were documented in Array 1b. It is feasible that a single pair hatched the first and third broods or second and fourth broods, however, the number of days between estimated hatch dates for the first and second broods and the third and fourth broods were 23 and 14 days apart, respectively. The breeding phenology within Array $1 \mathrm{~b}$ suggests breeding territories of two or more pairs overlapped within the camera array. Chicks from different broods were observed in close proximity to each other. On 30 August 2019, a ten day old chick from the fourth brood closely following an adult appeared in the same photograph as a partially feathered chick presumably from the third brood in the array. Chicks from both broods continued to be photographed in the array throughout September.

We documented 57 interactions between adult Black Rails and their chicks (Fig. 5). During these interactions, both adults $(n=7)$, a male $(n=22)$, a female $(n=12)$, or an adult of unknown sex $(n=16)$ were present. The most frequently observed chick-adult behaviors were chick(s) following an adult ( $n=$ 49), being brooded $(n=11)$, being fed $(n=7)$, and being preened $(n=1)$ (Fig. 5). We observed multiple behaviors during 14 interactions. Chicks solicited brooding and feeding by adults by bowing, fluttering their wings and vocalizing. Broods of downy chicks were frequently observed in a group sitting on the ground and preening themselves, and siblings occasionally pecked each other. Young continued to be observed within natal territories beyond fledging but were not observed receiving parental care after 25 days post-hatching. Although most chicks over 25 days old were alone in photographs, we observed two chicks from one brood brooding each other at 26 days and allopreening at 37,38 , and 39 days. Their allopreening behavior closely resembled allopreening courtship behavior between adults.

\section{Discussion}

Vocalization-based surveys are the predominant tool used to study secretive marsh birds (Taylor and van Perlo 1998; Conway 2011), but have a limited ability to determine basic biological characteristics, such as 
the phenology of incubation, brood rearing, and flightless molt. Camera trapping is emerging as a valuable tool for the study of rails (O'Brien and Kinnaird 2008; Colyn et al. 2017; Shirkey et al. 2017; Znidersic 2017; Colyn et al. 2019; Znidersic 2019), and has the ability to capture the chronological development of these characteristics. We successfully applied camera trapping techniques to the study of the Eastern Black Rail in coastal South Carolina and conducted the longest sustained sampling effort of any kind for the subspecies. Our results provide novel information about their nesting and breeding phenology, of which very little is known. Prior to our study, 146 egg date records spanning 137 years were available for the Eastern Black Rail (Watts 2016). Most nesting records for Black Rails come from historic accounts by egg collectors (Bent 1926), which do not include lay or hatch dates (Eddleman et al. 2020). We have contributed 33 hatch date records, more than any previous study of the species, and the only Atlantic coast records collected between 2010 and 2019 (Watts 2016).

Our breeding phenology estimates were based on photographic documentation of chicks in their respective broods rather than observations of eggs or nests. Chick growth and development in the Black Rail was virtually unknown prior to our study (Eddleman et al. 2020; U.S. Fish and Wildlife Service 2018), therefore we first needed to document the duration and progression of development from hatching to fledging before we could estimate hatch dates. In the only prior study of Laterallus chick development, Franklin et al. (1979) described physical development of Galapagos Rails (Laterallus spilonota) based on 11 chick captures, 10 juvenile captures, and 10 adult captures, however, in their study development was based on body weight rather than age post hatching, precluding direct comparisons of developmental timing. Chicks in our study exhibited a similar progression of development of eye and bill color compared to Franklin et al. (1979). During our study, we determined fledging occurs at approximately 40 days after hatching. This timeframe differs slightly from Eddleman et al. (2020), who noted that juvenile plumage is thought to be attained during the first six weeks while also acknowledging the timing of development was uncertain. While Black Rail eggs are currently thought to hatch synchronously (Walker 1941; Davidson 1992; Eddleman et al. 2020), we observed asynchronous development among siblings, demonstrating a small amount of variability in developmental rates.

The photographs and videos we captured of breeding behaviors of the Black Rail have provided valuable insights into their fecundity. Behavioral observations in conjunction with our phenology data suggested pairs successfully raised as many as three broods during a single breeding season and corroborate previous findings of Black Rails laying two or more clutches in one breeding season. Flores and Eddleman (1993) reported a female California Black Rail with an egg in her oviduct 18 days after her previous brood hatched and a male incubating eggs in both April and August. Second broods are not unusual in Rallidae (Taylor and van Perlo 1998) and have been reported in at least two of the remaining eight Laterallus species. Franklin et al. (1979) reported a banded Galapagos Rail that was associated with two distinct broods during a breeding season, and in captivity, Everitt (1962) observed a pair of Redand-white Crakes (Laterallus leucopyrrhus) raise two broods successfully. The apparent ability of the Eastern Black Rails to produce large clutches of eggs, nest throughout five months in the lower latitudes of their range, and successfully raise as many as three broods suggests the subspecies has the potential 
for high fecundity in all or part of their range (Todd 1977; Flores and Eddleman 1993; Legare and Eddleman 2001).

All North American rail species are temporarily flightless at the end of their breeding season during definitive prebasic molt (Pyle 2008). Prior to our study, the only observation of flightless molt in the Black Rail was a male California Black Rail captured in Arizona during September 1987 or 1988 (Flores and Eddleman 1991; Eddleman et al. 2020). Pyle (2008) asserts that North America Black Rail populations undergo definitive prebasic molt July through September, however the Eastern Black Rails observed in our study molted flight feathers during August through October. As evidenced by decreased body weights observed during late summer in California Black Rails (Flores and Eddleman 1991) and the high energetic and nutritional costs of flight feather replacement documented in species with similar molt strategies (Guillemette et al. 2007), adult Black Rails may be at increased risk of starvation and predation during this period if habitat conditions are not favorable.

The increased frequency and severity of flooding events resulting from sea level rise and climate change are among the greatest threats to the productivity and survival in tidal marsh-dependent avian species in the Eastern USA (Salt Marsh Bird Conservation Plan for the Atlantic Coast 2019). To understand and predict the responses of Black Rail populations to these threats, we must first recognize when these events coincide with periods of elevated vulnerability such as breeding and flightless molt. More than $75 \%$ of the remaining Eastern Black Rails in Atlantic and Gulf Coast states are believed to breed and molt in coastal Texas, Florida and South Carolina (Watts 2016). During the three years of our study, all areas known to be occupied by Black Rails in these states (U.S. Fish and Wildlife Service 2018) experienced expansive flooding resulting from tidal surge and/or rainfall associated with hurricanes on one or more occasions during the timeframe when we documented flightless molt in coastal South Carolina. These areas are also experiencing increasingly frequent and severe high tide flooding due to sea level rise. Sweet et al. (2020) reported that the frequency of high tide flooding in the U.S.A. doubled between 2000 and 2020, with Atlantic and Gulf Coast locations flooding at twice the national rate. We suggest continued and expanded monitoring of breeding and molt phenology using camera trapping techniques, which are relatively noninvasive compared to capturing and examining rails in hand, and are now proven in their ability to successfully monitor Black Rails. Our study provides novel resources that will facilitate the collection of phenology data in other areas of the Eastern Black Rail's range and the development of conservation strategies that maximize potential benefits to Eastern Black Rail populations.

\section{Declarations}

Competing interests: The authors declare no competing interests.

\section{ACKNOWLEDGEMENTS}

We thank the South Carolina Department of Natural Resources, the U.S. Fish and Wildlife Service, and private landowners for their logistical and/or financial support. Funding was primarily provided through 
the U.S. Fish and Wildlife Service Section 6 Grant Program (E-1-38, F17AP01001, and E-F19AP00004), State Wildlife Grant Program (SC-T-F17AF01208) and by the South Carolina Department of Natural Resources. We are grateful to the field technicians who spent countless hours maintaining camera traps and reviewing photographs. In particular, we thank K. Gundermann, S. Chandhok, C. Adams, C. Powell, C. Worthington, B. Donnelly and K. Brunk. SCDNR biologists and technicians D. Barrineau, J. Dozier, M. McAlister, J. Wood, K. Hiers, C. Cook, P. Snow, J. Bendt, Z. Simpson and J. Lemacks provided invaluable guidance and support. The Bear Island Wildlife Management Area, Yawkey Wildlife Center, and ACE Basin NERR provided housing. M. Legare, J. Wilson, W. Woodrow, W. Beisler, A. Smith, A. Schwarzer, and A. Tegeler generously shared their professional expertise and encouragement throughout the study. All applicable ethical guidelines for the use of birds in research have been followed, including those presented in the Ornithological Council's "Guidelines to the Use of Wild Birds in Research" (Fair et al. 2010). In addition, we thank J. Thibault, who provided valuable comments to improve previous drafts of the manuscript.

\section{Tables}

Due to technical limitations, the tables are only available as a download in the supplemental files section.

\section{Figures}




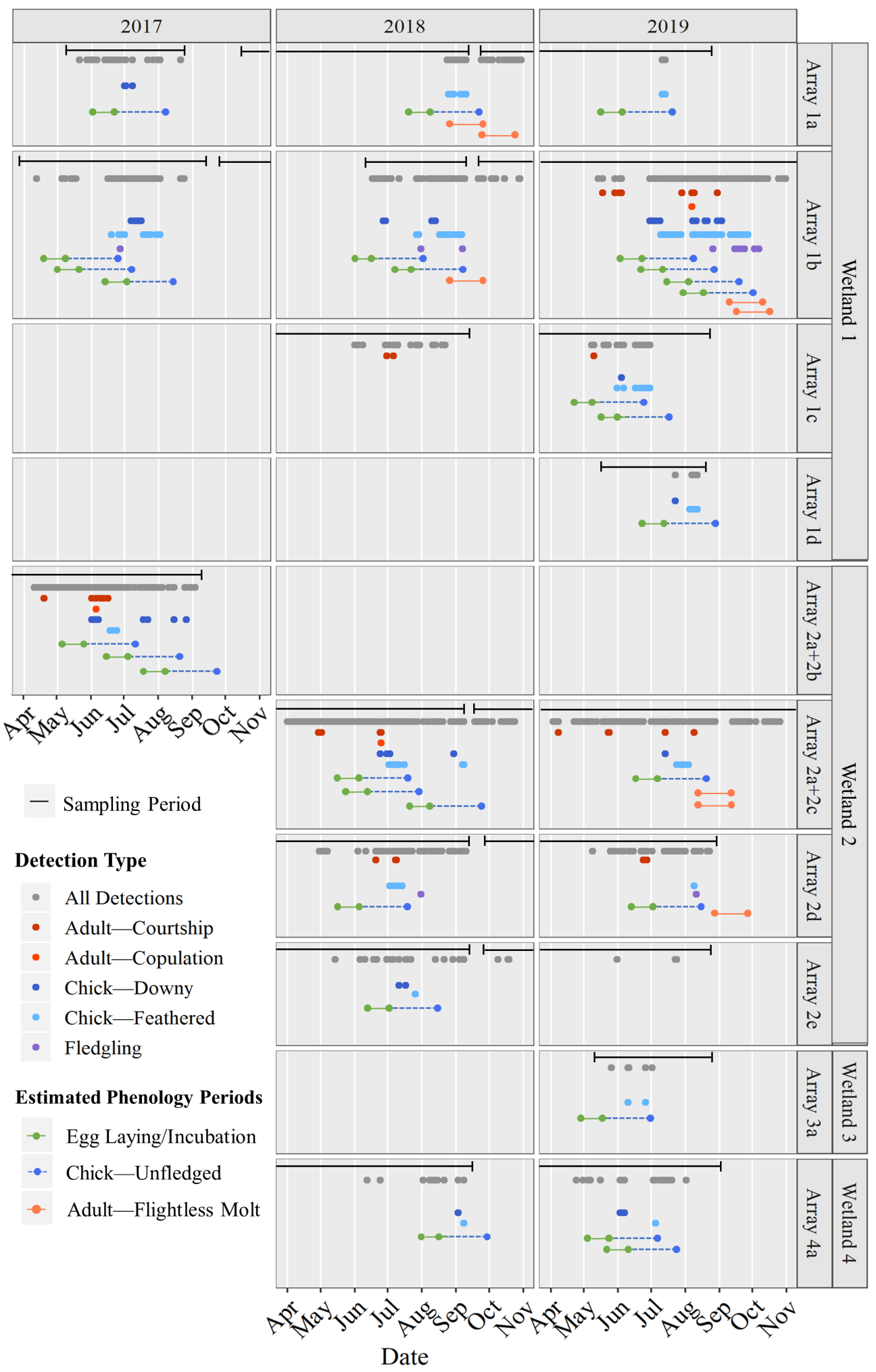

\section{Figure 1}

Phenology of breeding activity and flightless molt in the Eastern Black Rail (Laterallus jamaicensis jamaicensis) in Colleton and Georgetown Counties, South Carolina during 2017, 2018 and 2019. Observations collected using camera traps during varying sampling periods (black) were categorized as all life stages (gray), adults (red/orange), unfledged chicks (blue; downy from 1 - 15 days and feathered from 16 - 39 days) or fledglings (purple). Phenology periods were approximated based on detections of 
chicks and on an egg laying/incubation period of 25 days, unfledged chick period of 40 days, and flightless molt period of 21 days. Portions of Wetland 1 (Arrays $1 \mathrm{a}, 1 \mathrm{~b}$, and $1 \mathrm{~d}$ ) were burned during prescribed fires on 28 February 2018 and 26 February 2019.
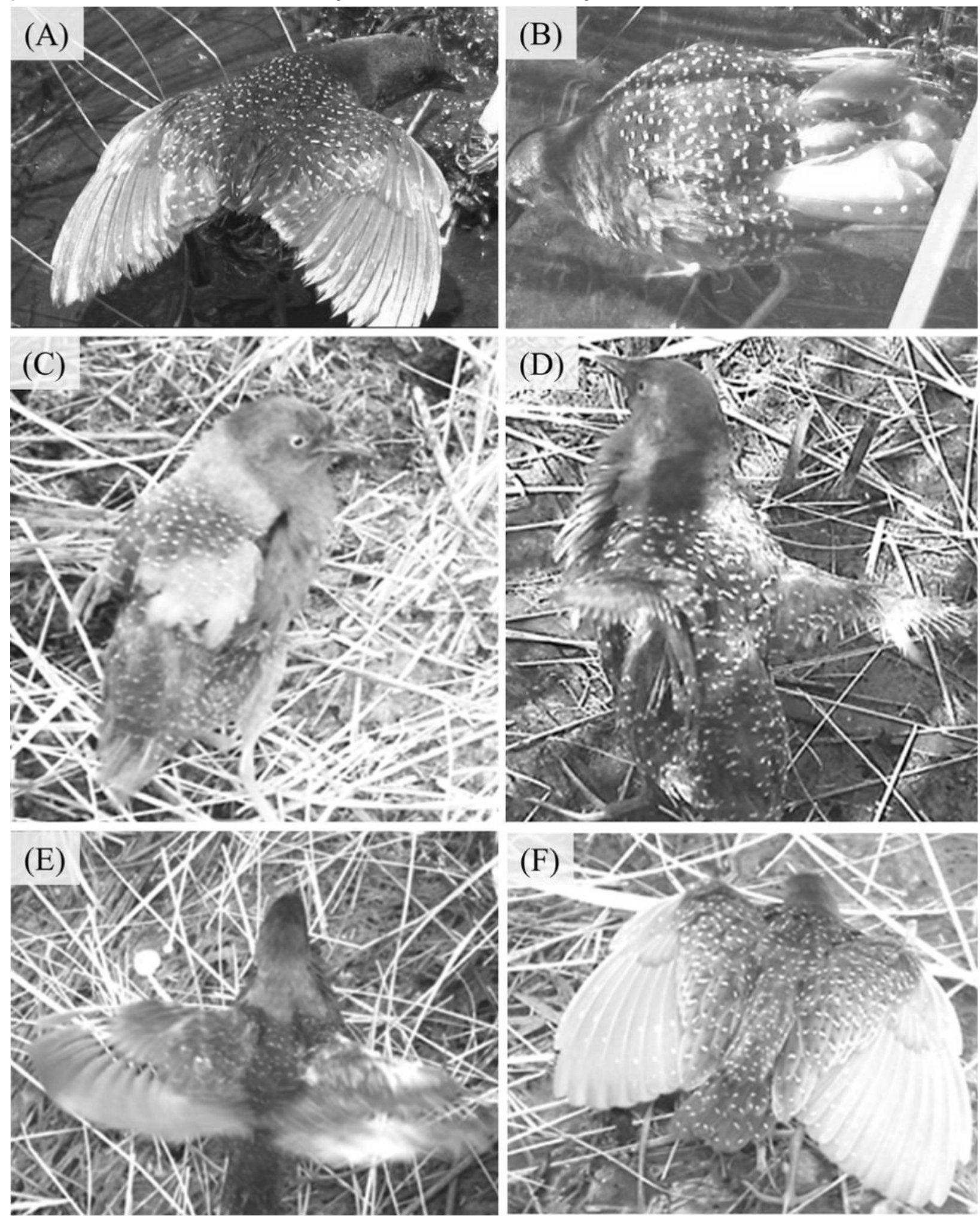

Figure 2

Progression of remige and retrice replacement in adult Black Rails (Laterallus jamaicensis jamaicensis) during definitive prebasic molt as documented using camera traps in Colleton County, South Carolina 
during August - October 2016, 2018, and 2019. Worn feathers (A) are lost simultaneously beginning on day 1 (B), and by day 3 (C) all feathers are lost, leaving adults unable to fly. Replacement feathers gradually emerge from sheathes ( $D$; day eight) and unfurl ( $E$; day 17$)$. By day 21 , feather replacement is complete (F; day 22) and adults are once again capable of flight. Photographs C, D, E and F were taken in a single camera trap array where an adult male was documented molting from approximately 16 September through 7 October 2019.

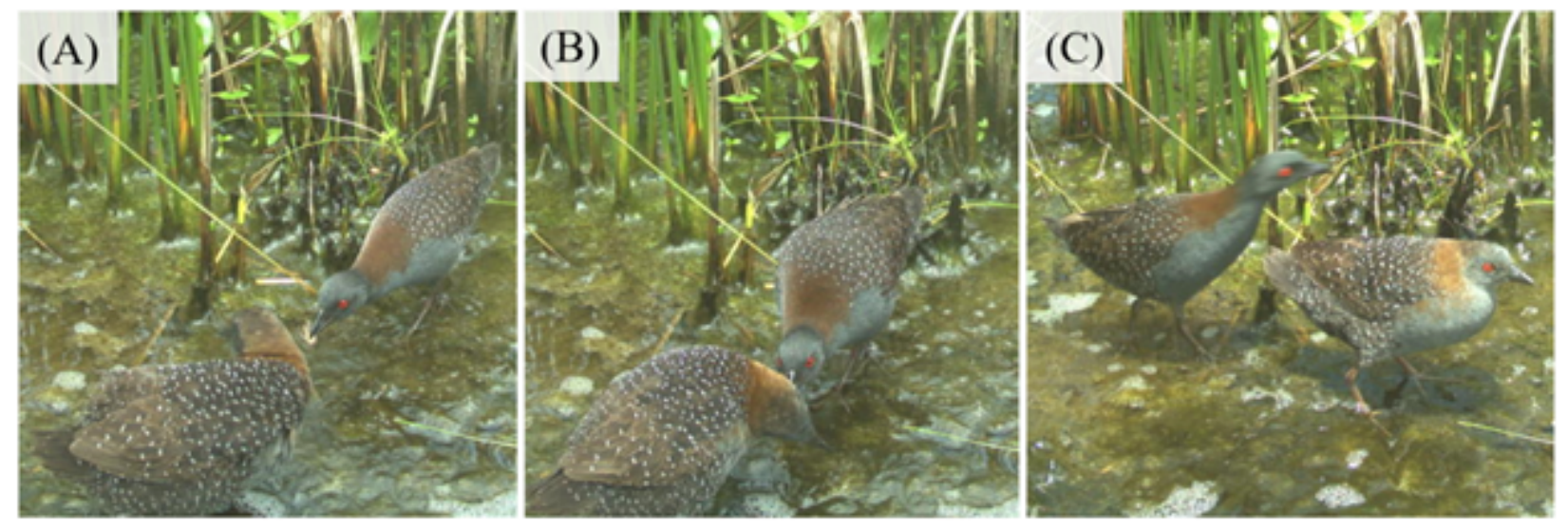

\section{Figure 3}

Courtship behaviors of the male (upper) and female (lower) Eastern Black Rail (Laterallus jamaicensis jamaicensis), including (A) offering food, (B) allopreening, and (C) pursuit of the female by the male documented using camera traps in Colleton and Georgetown Counties, South Carolina.

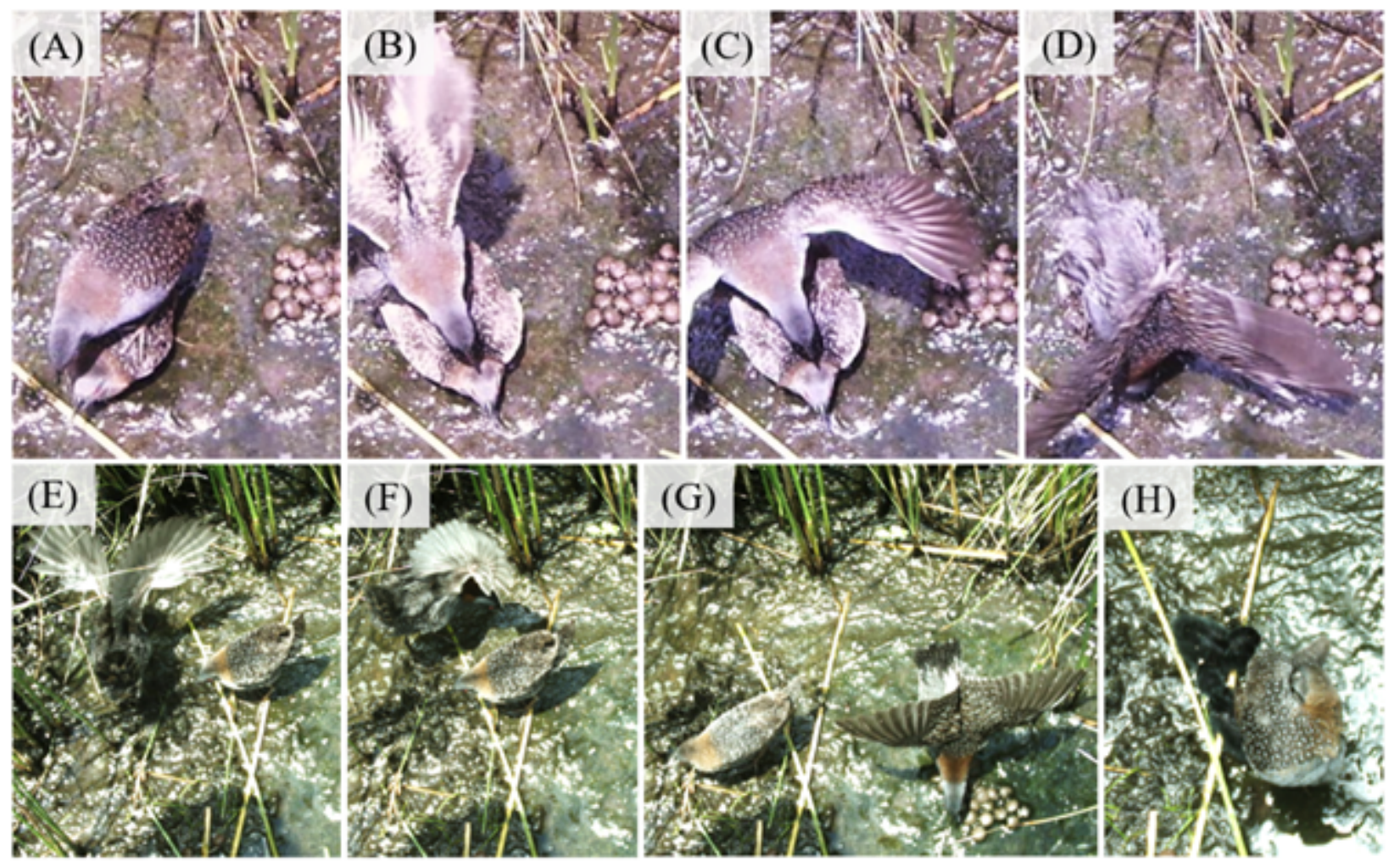

Figure 4 
Progression of copulation behaviors of the Eastern Black Rails (Laterallus jamaicensis jamaicensis) documented on 5 June 2017 in a tidal marsh in Colleton County, South Carolina. Photographs and video by camera traps show a male mounted a female at 12:50 (A), copulated (B-C), tumbled from the female (D), then circled the female with a lowered head and raised tail as the female bowed forward (E-G) before both adults departed from the field of view of the camera. At 13:43, an adult male was photographed with a brood of approximately five-day old chicks $(\mathrm{H})$ in the location where the copulation occurred. A female was photographed in this location with the chicks at 14:17.
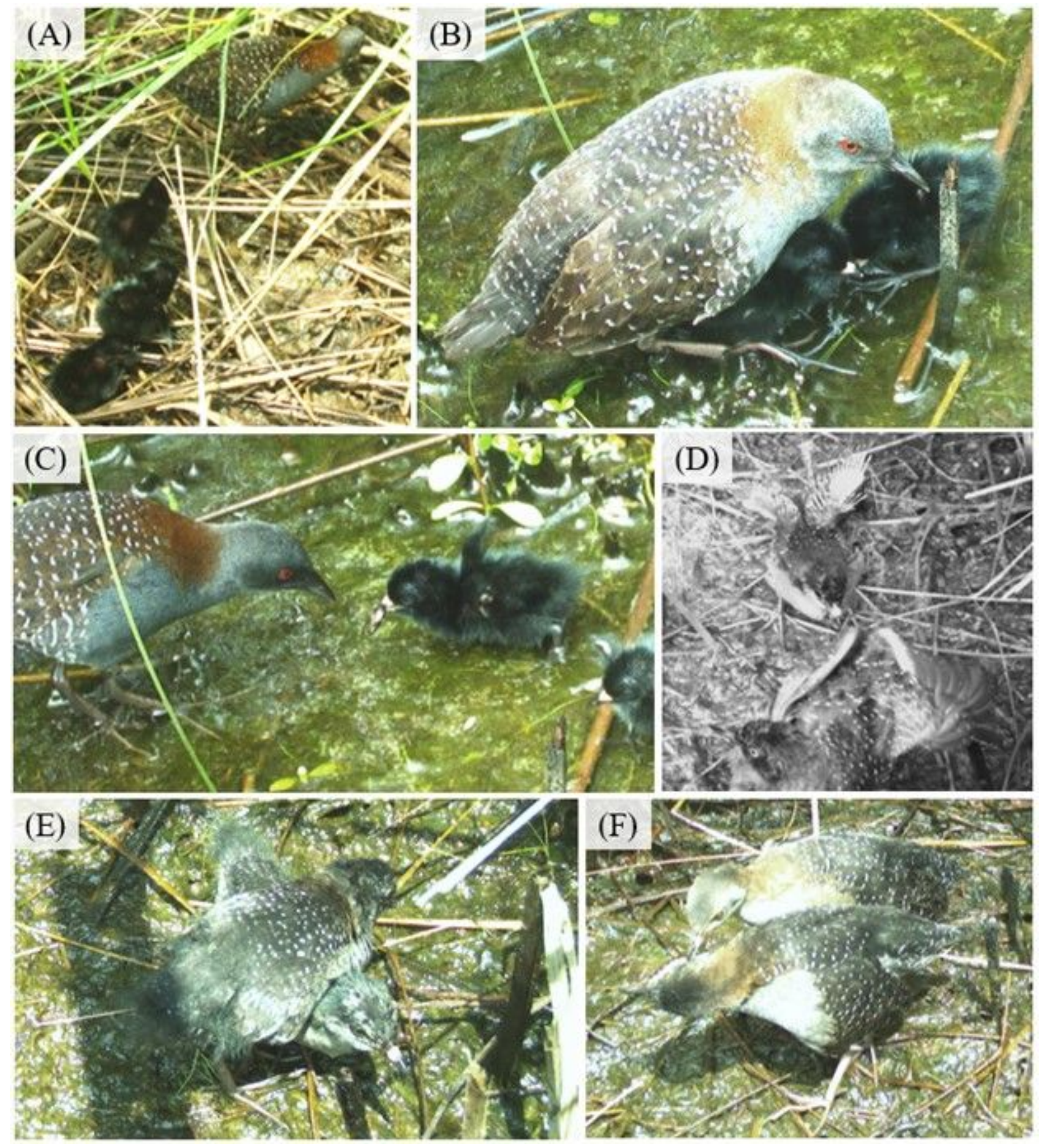

Figure 5 
Parental care and intrabrood interaction behaviors by Eastern Black Rails (Laterallus jamaicensis jamaicensis) observed in wetlands in Colleton County, South Carolina during 2017-2019. Male and female adults provided parental care by leading (A), brooding (B), and feeding chicks (C). Chicks fluttered their wings (D) and vocalized to solicit feeding and brooding from adults. Intrabrood interactions typically involved sitting on the ground or preening in close proximity; however, within one brood, we observed two chicks brooding each other $(E)$ and allopreening $(F)$.

\section{Supplementary Files}

This is a list of supplementary files associated with this preprint. Click to download.

- SCDNRSupplementalVideoCopulationDisplayBLRA.avi

- SCDNRSupplementalVideoParentalCareBroodingBLRA.mp4

- SCDNRSupplementalVideoAsynchronousDevelopmentParentalCareBLRA.mp4

- Tables.pdf 